\title{
ENVIRONMENTAL FACTORS AND THEIR EFFECT ON THE OCCURRENCE OF AUTISM
}

\author{
Varvara Tsopanidou ${ }^{1}$, Athanasios Drigas ${ }^{1}$ \\ ${ }^{1}$ Net Media Lab Mind - Brain R\&D IIT - N.C.S.R. "Demokritos", Athens, Greece
}

\begin{abstract}
The etiology of autism is the subject of intense research. Genetic factors are clearly important. Gene mutations, gene deletions, copy number variations (CNVs) and other genetic abnormalities are strongly linked to autism. But no etiology represents more than a relatively small percentage of cases. Environmental factors in recent years are increasingly coming to the forefront of research and study and the interest of researchers is increasingly directed in this direction. This article examines the environmental factors associated with the prenatal period and their relationship to autism.
\end{abstract}

Keywords: (environ)mental factors, neurodevelopment, toxicity, industrial exposure, autism, lead, mercury, dental, aluminum, mmr, heavy metals.

\section{INTRODUCTION}

Autism is a complex, serious, biological disorder based on impaired brain development, first described in 1943 by Kanner. Social deficits, communication disorders, repetitive behaviors and mental lack of flexibility are the main characteristics. Based on research, there is no specific biochemical marker or distinct neuroanatomical abnormality that defines autism, and the diagnosis is based on clinical evaluation and behavior.

When we want to find the etiology, there is not a single cause but many theories that try to explain the onset of autism. These causes could take many forms, such as defective genes, chromosomal abnormalities, metabolic disorders, infectious viruses. Any possible cause could affect the vital system involved in autism, whether or not it affects other systems (Frith, 1994). Developments in genetics identify the link between genes and autism, while also investigating whether exposure to chemicals "enhances" autistic behavior. Indirect evidence for the "triggering" of autistic behavior due to exposure to chemicals comes from studies that demonstrate the body's sensitivity to substances such as lead, ethyl alcohol and methylmercury (Landrigan, 2010).

For more than a century, autism has been believed to be a genetic and inherited disease (Chamak, 2010; Hallmayer et al., 2011; Landrigan, 2010; Tammimies et al., 2015). Recently, Hallmayer et al. (2011) conducted one of the largest studies with twin couples. They completed 192 studies with twin couples and reported that a higher risk of ASD in monozygotic twins was due to environmental factors and a lower risk was due to heredity or genetics.

Support for the possibility of an environmental contribution to the etiology of autism comes from two important sources: (1) the modern understanding of the exceptionalvulnerability of the developing human brain to toxic exposures to the environment and (2) significant studies that specifically demonstrate and link autism to environmental exposures experienced prenatally. (Landrigan, 2010).

\section{METHODOLOGY}

This study was based on the literature review of articles on the impact of environmental factors on the prenatal period and the occurrence of autism. For this literature review, articles were selected based on the following criteria: to include in the study the environmental factors related to the occurrence of autism before birth, such as toxic, chemical factors and heavy metals. The study excluded articles related to gene mutations, chromosomal abnormalities, genetic deficiencies, hormonal disorders, drug use. 
This review highlights the role of environmental factors in the development of autism in the context of emerging genetic research suggesting that brain synapses are an organ particularly vulnerable to genetic disorders and possibly environmental-related disorders. In particular, recent data suggest that immune system abnormalities and impaired zinc homeostasis may affect synaptic transmission. Understanding how genetic and environmental risk factors for autism converge in synapses can be a valuable starting point for future work to uncover the pathogenesis of autism.

\section{EPIDEMIOLOGY - FREQUENCY}

The frequency in most studies seems to be around 4-10 autistic children in every 10,000 births. But Wing \& Gould (1979) reported an incidence of 21 autistic children in every 10,000 for "the triad of social, language, and behavioral deficiencies" in Camberwell's study. Gillberg et al. (1986) found similarly high rates of trinity and mental retardation in Swedish adolescents.

Other studies report a frequency of around 10 autistic people per 10,000. These recent studies (from America, Japan and France respectively) show that autism is ubiquitous in the world and is less common in some societies than in others. While the reported incidence of autism has increased in recent years, this is probably due to better information and a broader perception of autism (Schopler \& Mesibov 1987).

All epidemiological studies show a significantly higher number of boys than girls with autism. The ratio of boys and girls varies from 2: 1 to almost 3: 1 . The sex ratio seems to vary by ability: most autistic girls are at the lower end of the capacity range, while at the upper end of the capacity ("Asperger syndrome") boys may outperform girls by a 5: 1 ratio. . Szatmari \& Jones (1991) have suggested some possible reasons for lower IQ of autistic women. For example, women may be more strongly influenced by the autism gene, or there may be genetic heterogeneity with "milder" forms (incomplete penetration) of the X chromosome-related disorder, which is why it is more common in boys (Mesibov, Lynn, Klinger, 1997).

\section{CAUSES OF AUTISM}

The reason for the ASD has not yet been clarified. Candidate causative agents include genetic, neuroanatomical, environmental, immune and pathological conditions, which interact and contribute to different way and degree in the onset and progression of these disorders. Studies show that in at least $10 \%$ of people with autism, autistic behavior accompanies a monogenic disease or syndrome, such as Fragile X syndrome, Prader-Willi / Angelman syndrome, Sclerosis, Phenylketonuria, Neuroinoma and mitochondrial diseases. Interestingly, according to research, $1.7-4.8 \%$ of the total have a variety of chromosomal abnormalities, which may reflect the fact that the disorder of several genes leads to a nonspecific brain damage whose common final expression is autism. (Volaki, K. 2012 ).

\section{GENETIC FACTORS AND AUTISM}

Autism is one of the most inherited disorders in Psychiatry. Despite the high degree of heredity, the etiology is heterogeneous, with possible involvement of many genes and chromosomal regions and the influence of environmental factors that add to the strong genetic predisposition. Genetic heterogeneity goes hand in hand with the diversity of the clinical picture. Each gene probably contributes in a different way to the onset of the disorder (even different variants of the same gene). Research in people with autism provides strong evidence that ten or more genes interact to cause autism and that different groups of genes may be responsible for different families. (August, Stewart, \& Tsai, 1981. Szatmari \& Jones, 1991)

\section{EPIGENETIVE FACTORS}

Since the genetic code does not provide an explanation for the onset of autism, its etiology may also involve non-genetic factors of epigenetic and environmental origin. Epigenetic changes such as DNA methylation, RNA silencing and histone modification can lead to improper expression or silencing of genes critical to brain development. (Volaki, K. 2012) 


\section{ENVIRONMENTAL FACTORS ACCUSED OF AUTISM}

\section{Toxic agents and autism}

The fetal brain is a complex, sensitive organ, made up of billions of cells where it is created and developed under a very narrow and specific time frame during pregnancy. This sensitivity makes him extremely vulnerable when exposed to toxic agents compared to a similar exposure of an adult brain. The fetal blood-brain barrier is not fully formed until the sixth month of pregnancy, leaving the brain immune to toxins such as heavy metals that can enter the fetus's body through the mother's blood. Even after the baby is born, the brain remains sensitive and fragile as it is unable to detoxify exogenous and lipophilic substances that can easily pass into it through breast milk.

Studies have shown that $1 / 6$ of children in the US suffer from developmental dysfunction where in most cases it is responsible for this nervous system. The US National Research Council states that about 3\% of developmental disorders are the direct result of environmental pollutants, while $25 \%$ result from the interaction of environmental factors and the sensitivity of the human gene. (Depastas Ch. 2017). Biomarkers are substances that are detected in the blood and urine. In children with autism biomarkers are usually at lower levels, such as antioxidant enzymes, omega-3 fatty acids, folic acid, vitamin B1 and probiotics. Another biomarker is oxidative stress, which is caused by environmental toxins and toxic substances such as heavy metals, mercury, pesticides, maternal infections, and mitochondrial dysfunction, which has been implicated in the etiology of autism. (Theodora Stavridou, Anna Maria Driga, Athanasios Drigas)

\section{Chemical agents}

In a unanimous scientific report of the Collaborative on Health and the Environment's Learning and Developmental Disabilities Initiative, several environmental factors were identified as powerful factors in creating learning and developmental difficulties in humans. These included arsenic, lead, manganese, mercury, pesticides, biphenyl ethers (PBDEs), polychlorinated biphenyls (PCBs), polycyclic aromatic hydrocarbons (PAHs), and solvents.

Some possible sources of heavy metal poisoning include chemicals, fertilizers, industrial paints, building materials, high mercury fish, silver dental fillings, and mercury-containing preservatives (thiomersal) in vaccines. Lead can be found in dirt near roads and can even be found in house paints. Children who eat pigmented chips may develop toxic lead levels. Genetically, children with a predisposition to autism may be less able to detoxify toxic environmental factors, and this inability may predispose them to nerve damage and to develop autistic behavioral characteristics. (Geier et al. 2009)

\section{Heavy metals}

Heavy metal contamination is ubiquitous and children are generally more susceptible to heavy metal toxicity than adults, but exposure to heavy metals received in early development is difficult to distinguish after being diagnosed with autism. Nevertheless, several studies have evaluated the correlations between autism and biomarkers of exposure to heavy metals and their effects. Evidence that exposure to heavy metals leads to an autism phenotype is becoming increasingly convincing. (Dietert, R. R., et al. 2011). Sulfhydryl metals such as arsenic, cadmium, lead and mercury are metals commonly referred to as associated with the onset and risk of autism. (Gilbert SG).

Kern et al. evaluated hair samples from autistic and control children and found that sulfhydrylreactive metal levels were lower in autistic children than in control children, suggesting that subgroups of autistic children may be hypersensitive to certain metals because they are low in metal detoxification. Other metals may also increase the risk.

Adams et al. reported that the severity of autistic symptoms was affected by body loads of aluminum, antimony, lead, and mercury, as measured by urinary excretion. Recent data on mercury toxicity related to autism biology are more convincing as Palomo et al., 2003; Ashner and Walker, 2002; Bernard et al., 2002; Vojdani et al., 2003 and Bradstreet et al. (2003) report that urinary mercury levels after 3 days of oral chelating agent, meso-2,3-dimercarctosuccinic acid (DMSA), in children with autism spectrum disorders were three times higher than in a corresponding standard control sample. 
The risk of developmental toxicity with low-dose chemicals is likely to be influenced by individual genetic susceptibility determinants such as the frequency of polymorphic variants affecting tissue distribution and the toxicity of lead and mercury. The extreme sensitivity of the developing brain and immune system to very low levels of lead and mercury gives this hypothesis biological credibility. (Rose et. All. 2008). Hypersensitivity to environmentally significant levels of lead and mercury due to individual genetic variants is a reasonable hypothesis that could potentially lead to neurodevelopmental toxicity and increase the risk of autism. Both lead and mercury cause oxidative damage by eliminating intracellular glutathione, the major intracellular mechanism for heavy metal detoxification.

\section{Mercury}

Mercury is reported as the third most common (arsenic and lead are the first and second) toxic substances in the United States (ATSDR, 2001). The symptoms of nervous system disorders associated with chronic exposure to mercury have been known since the 19th century, when mercury was widely used in the felt industry leading to the expression "hat disease" (Hu, 1998).

Exposure to heavy metals has been linked by many studies to autism. Early research looked at methylmercury $(\mathrm{CH} 3 \mathrm{Hg})$ where it was found that it can impair normal brain development by creating a range of neurodevelopmental disorders. Its highest levels have been found in large fish where it is the main source of human exposure. (Geier DA, King PG, Sykes LK, Geier MR).Other forms of exposure to mercury are from the use of medicines (eyes, ears, nose, throat, skin), creams, perfumes, cosmetics, vaccines, allergy tests, antiseptics, disinfectants, fungicides, herbicides, thermometers, dentistry and more. In addition, a study reports that levels of mercury, lead, and zinc in autistic baby teeth were on average twice as high as in non-autistic babies.

The survey is reported as statistically insignificant. The greatest exposure to mercury is from the environment. The incidence of autism is increasing in areas with high levels of heavy metals such as carbon, lead and mercury (burning areas) in the atmosphere - high levels of air pollution. (Rossignol DA, Genuis SJ, Frye RE / - Van Wijngaarden E, Davidson PW, Smith TH, Evans K, Yost K, Love T, et al. )

A Texas ecological study found an association between the release of environmental mercury into the atmosphere and autism (Palmer et al., 2006). Transmission from mothers to infants is well documented in animal models (Newland et al., 1994) but also in human studies (Ramirez et al., 2000; Grandjean et al. 1995). The results of various studies show that maternal mercury exposure during pregnancy is associated with neuropsychological deficits in children and that this association is more evident in women with constant exposure throughout pregnancy. (Ramirez et al., 2003; Grandjean et al., 2003).

\section{Thalidomide}

Thalidomide, which was known to cause genetic abnormalities when used as an antiemetic in pregnancy in 1950/1960, is still of pharmacological interest given its immunomodulatory effects (Kumar and Chhibber, 2011). The observed immunomodulation by thalidomide may be associated with an increased risk of prenatal infection. Thalidomide, for example, regulates cytokine levels and macrophage proinflammatory function (Kumar et al., 2010). It has also been found to have anti-angiogenic and immunomodulatory properties including $\mathrm{T}$ cell co-stimulation, and activation of NK cells (natural killer) (Quach et al., 2010). The incidence of autism is significantly higher in children who are prenatally exposed to the anticonvulsant factor valproic acid (Moore et al., 2000) or the immunomodulatory factor thalidomide (Stromland et al., 1994) at the beginning of the first trimester of pregnancy.

\section{Valproic acid}

Children who are prenatally exposed to the anticonvulsant valproic acid show patterns of physical malformations similar to those of thalidomide embryopathy, but less severe. These include neural tube defects, heart malformations, facial skull abnormalities, and limb defects.They may also develop autism (Moore SJ, Turnpenny P, Quinn A, et al.). Autism was reported in $11 \%$ of the 57 children whose mothers received valproic acid early in pregnancy. (Snow WM, Hartle K, Ivanco TL.)

\section{Misoprostol}

Misoprostol is an analogue of prostaglandin, which is licensed in the United States to prevent gastric ulcers. It is widely used in some countries for abortions.A number of cases from Brazil describe a group 
of seven children with ASD, of whom four (57.1\%) had prenatal exposure to misoprostol (Bandim JM, Ventura LO, Miller MT, et al.) Relevant reports reported in the first trimester of pregnancy after unsuccessful abortion attempts. The average exposure was six weeks after arrest.

\section{Bromides (pbde) and autism}

Existence levels of brominated retardants (BFRs) in the environment have increased significantly in recent years. Levels found in breast milk and fetal blood are also high worldwide. Compounds in this category can and do act as hormone mimics even when structural similarities are not immediately apparent.

Polybromodiphenyl ethers (PBDE) are chemical flame retardants found in electronic products, textiles, etc. They are chemical analogs of thyroid hormones, which enable them to regulate the exact time of regulation of brain cell growth. Polybromodiphenyl ethers (PBDEs) attach to the protein-carrying thyroid hormone and can also interact with androgen and estrogen receptors. The pregnant woman can be exposed through food, dust, etc. thus affecting the developing brain of the fetus. (Depastas. 2017) The affinity for PBDE in human breast milk from Swedish women has increased exponentially in the last two decades (Sjodin A. et al., Lind Y. et all) and studies in US populations have shown the presence of PBDE in human breast milk, adipose tissue and blood. (Schecter A. et all., Betts KS.) Interestingly, PBDE levels in U.S. women's breast milk reflect a body load far greater than that reported in the Nordic studies. (Sjodin A. et all ., Schecter A. et al.) Specifically, PBDE levels in women in Northern California are among the highest levels reported to date, as expected for the San Francisco Bay Area, one of the most polluted areas in the world. PBDE levels in breast fat from women living in this area were 3 to 25 times higher than in other parts of the world. (Wong, S. et all 2016)

\section{Chloropyrifos}

It is an organophosphate insecticide that was widely used until a few years ago in schools, homes and agriculture. A study conducted in California (Roberts et al. 2007) aimed to key periods of pregnancy may be associated with the development of Autism Spectrum Disorders (ASD) in children. The risk of ASD was consistently associated with homes close to pesticide applications in organochlorides occurring around the CNS embryogenesis period.

Attention deficits were reported at the ages of 36 months and 5 years. Lower IQ scores and reduced functional memory were reported in different studies over 7 years. A more recent study found a positive correlation between maternal and dimethyl phosphate during pregnancy and reported maternal measurements and executive functions in children aged 6-9. (Lan, A. et al.2019).

The results of the study are in line with the results of Engel et al., Who found a similar deficit in neuromotor development in neonates who exhibited abnormal endometrial reflexes and premature postpartum exposure to organophosphates. Infants at high risk or later diagnosed with Autism Spectrum Disorders (ASD) showed more deficits in reflexes and spontaneous motor movement during childhood than children usually develop. While retarded reflexes are not specifically related to the autism spectrum, delays in achieving milestones of motor skills, abnormal reflexes, and posture asymmetries have been reported to occur in infants and young children who were later diagnosed with ASD. (Lan, A. et al. 2019).

\section{Synthetic chemicals}

Over the past 3 decades, a significant increase in the prevalence of autism has been reported, from 4 to 5 per 100,000 in the 1960s to about one in 45 children today (Zablotsky et al., 2015). It is argued that the alarming increase in autism in recent years is due to the human population being exposed to an increasingly diverse set of synthetic chemicals, including perfumes, many of which contain steroids (a male and female type of chemical hormone (Sarantis et al.)., 2010).

According to published laboratory and epidemiological studies, exposed chemicals in perfumes, such as those that lead to different odors, increased shelf life, control the release time of perfumes and improve stability, have endocrine disrupting properties. (AbramssonZetterberg and Slanina, 2002; 
Gomez et al., 2005; Inui et al., 2003; Kunz et al., 2006; Liu et al., 2009; Reiner et al., 2007; Roy et al., 2009; Sarantis et al., 2010; Schmutzler et al., 2004; Spencer et al., 1979; Suzuki et al., 2009; Swan, 2008; Furuhashi et al., 1994). Many synthetic fragrances are believed to contain testosterone hormones (Bagasra et al., 2013; Sarantis et al., 2010.)

Shaw et all. (2014a) and Shaw et al. (2014b) claim that neurodevelopmental disorders, such as autism, are caused by abnormal immune signaling activity during fetal development, which is likely to be caused by exposure to xenobiotics (Shaw et al., 2014a). In addition, they suggest that pregnant women and infants are exposed to xenobiotics worldwide, such as mercury and aluminum, through pediatric vaccinations (Shaw et al., 2014b).

\section{CONCLUSIONS - DISCUSSION}

Environmental chemical exposures are becoming increasingly understood as important in causing autism, and current theories suggest that autism is caused by the interaction of multiple genetic and environmental contributions that vary from person to person. Although 70 the original studies suggested a strong genetics

Inheritance of autism, recent studies with larger sample sizes have shown less impact, including a study of more than 14,000 children with autism in Sweden who showed 50\% heredity, supporting an equally strong role for environmental risk factors. (Kalkbrenner et al. 2014)

For this reason, articles have been published that study the relationship between brominated retardants (BFR), bisphenol A (BPA), chlorophyll organophosphate insecticide, thalidomide, which was used for anxiety but also for diseases of pregnancy and the ulcer but also the induction of abortions. (Kalkbrenner et al. 2014) Diet is a predominant source of exposure to many persistent pollutants (PCBs, PBDEs and PFCs) because they are bioaccumulated in fish and breast milk. In addition, these chemicals can be widely present indoors because the chemicals are not bound by the products in which they are used and thus are easily released into the environment, leading to further ingestion or inhalation. Examples include PBDEs in indoor powders after release from computers or carpets and phthalates released from perfumes and scented products. All of these chemicals, whether banned or not, whether they are persistent or not, are found almost worldwide in representative human biological samples from the United States.

The potential developmental neurotoxicity of heavy metals that is often encountered in the environment is an important public health issue and challenge, because their identification and regulation could lead to preventive measures. Grandjean and Landrigan elegantly addressed this issue in a recent review and provided evidence of the many untested and uncontrolled chemicals in drinking water, soil and air that may contribute to the development of neurodevelopmental disorders in modern society. For example, environmentally significant lead levels have been shown to be associated with cognitive and developmental deficits that translate into long-term deviations in attention, impulsivity, aggression, and 71 in impaired IQ, memory, and language skills. (Tellez-Rojo et al.2006- Lanphear et al. 2005)

Additives and synergistic interactions between chemicals and differences in duration, time, and dose during critical developmental stages complicate the retrospective evaluation of neurodevelopmental toxicity (Rice, Barone 2000). Future evaluation of maternal and umbilical cord blood, as proposed in the National Pediatric Study, could provide valuable information on early toxic exposure and the consequent risk of developmental disabilities. An alternative approach is to evaluate genetic polymorphisms that could give differential sensitivity to specific environmental reports. (Rose et all.2008)

Grandjean and Landrigan (2006) hypothesize that our exposure to chemicals that have not been adequately tested for developmental neurotoxicity has led to a silent pandemic. Further research is needed to provide the evidence base that can ultimately lead to the reduction or elimination of these potentially harmful reports through changes in regulatory policy, consumer behavior or dietary choices. (Shelton et al. 2012)

To discover the unexpected environmental causes of autism, an interdisciplinary autism detection strategy is proposed that combines toxicological testing, neurobiological research, and future epidemiological study. There is a possibility that this strategy will identify new environmental causes of autism, causes that can theoretically be avoided. The potential for discovery is great. (Landrigan, 2010) 


\section{References}

[1] Adams JB, Baral M, Geis E, Mitchell J, Ingram J, Hensley A, et al. The severity of autism is associated with toxic metal body burden and red blood cell glutathione levels. J Toxicol. 2009: 532640.

[2] Bandim JM, Ventura LO, Miller MT, et al. Autism and Mo"bius sequence: an exploratory study of children in northeastern Brazil. Arq Neuropsiquiatr 2003; 61 (2A):181-185.

[3] Chamak, B. (2010). Autism: overestimation of the genetic origins. Medecine Sciences: M/S, 26(67), 659-662.

[4] Charalambos, D. (2017). Toxic agents and autism. Rostrum of Asclepius / Vima tou Asklipiou, $16(3)$.

[5] Dietert, R. R., Dietert, J. M., \& DeWitt, J. C. (2011). Environmental risk factors for autism. Emerging health threats journal, 4(1), 7111.

[6] Frith, U., Happe, F., \& Siddons, F. (in press). Theory of mind and social adaptation in autistic, retarded and young normal children. Social Development.

[7] Geier DA, King PG, Sykes LK, Geier MR. A comprehensive review of mercury provoked autism. Indian J Med Res 2008; 128:383-411.

[8] Gilbert SG (Institute of Neurotoxicology and Neurological Disorders). Scientific consensus statement on environmental agents associated with neurodevelopmental disorders. Collaborative on Health and the Environment's Learning and Developmental Disabilities Initiative; 2008 July 1 [accessed 2010 July 6]. Available from: http://www.iceh.org/pdfs/LDDI/ LDDIStatement.pdf

[9] Grandjean, P., Weihe, P., \& White, R. F. (1995). Milestone development in infants exposed to methylmercury from human milk. Neurotoxicology, 16(1), 27-33.

[10] Hallmayer, J., Cleveland, S., Torres, A., Phillips, J., Cohen, B., Torigoe, T., ... \& Risch, N. (2011). Genetic heritability and shared environmental factors among twin pairs with autism. Archives of general psychiatry, 68(11), 1095-1102.

[11] Kalkbrenner AE, Daniels JL, Chen JC, Poole C, Emch M, Morrissey J. Perinatal exposure to hazardous air pollutants and autism spectrum disorders at age 8. Epidemiology. 2010;21:631641.[PMC free article] [PubMed] [Google Scholar]

[12] Kern JK, Grannemann BD, Trivedi MH, Adams JB. Sulfhydryl-reactive metals in autism. J Toxicol Environ Health A. 2007;70:71521

[13] Kumar, V., \& Chhibber, S. (2011). Thalidomide: an old drug with new action. Journal of Chemotherapy, 23(6), 326-334.

[14] Kumar, V., and Chhibber, S. (2011). Thalidomide: an old drug with new action. J. Chemother. 23, $326-334$.

[15] Kumar, V., Harjai, K., and Chhibber, S. (2010). Thalidomide treatment modulates macrophage pro-inflammatory function and cytokine levels in Klebsiella pneumoniae B5055 induced pneumonia in BALB/c mice. Int. Immunopharmacol. 10, 777-783.

[16] Lan, A., Stein, D., Portillo, M., Toiber, D., \& Kofman, O. (2019). Impaired innate and conditioned social behavior in adult C57B16/J mice prenatally exposed to chlorpyrifos. Behavioral and Brain Functions, 15(1), 2.

[17] Landrigan, P. J. (2010). What causes autism? Exploring the environmental contribution. Current opinion in pediatrics, 22(2), 219-225

[18] Lind Y, Darnerud PO, Atuma S, Aune M, Becker W, Bjerselius R, et al. Polybrominated diphenyl ethers in breast milk from Uppsala County, Sweden. Environmental Research. 2003; 93(2):186194. [PubMed: 12963403]

[19] Moore SJ, Tumpenny P, Quinn A, Glover S, Lloyd DJ, Montgomery T, et al. (2000): A clinical study of 57 children with fetal anticonvulsant syndromes. J Med Genet 37:489-497.

[20] Moore SJ, Turnpenny P, Quinn A, et al. A clinical study of 57 children with fetal anticonvulsant syndromes. J Med Genet 2000; 37:489-497. 
[21] Palmer, R. F., Blanchard, S., Stein, Z., Mandell, D., \& Miller, C. (2006). Environmental mercury release, special education rates, and autism disorder: an ecological study of Texas. Health \& Place, 12(2), 203-209.

[22] Quach, H., Ritchie, D., Stewart, A. K., Neeson, P.,Harrison, S., Smyth,M. J., et al. (2010). Mechanism of action of immunomodulatory drugs (IMiDS) in multiple myeloma. Leukemia 24, $22-32$.

[23] Ramirez, G. B., Cruz, M. C. V., Pagulayan, O., Ostrea, E., \& Dalisay, C. (2000). The Tagum study I: analysis and clinical correlates of mercury in maternal and cord blood, breast milk, meconium, and infants' hair. Pediatrics, 106(4), 774-781.

[24] Roberts, E. M., English, P. B., Grether, J. K., Windham, G. C., Somberg, L., \& Wolff, C. (2007). Maternal residence near agricultural pesticide applications and autism spectrum disorders among children in the California Central Valley. Environmental health perspectives, 115(10), 1482-1489.

[25] Rose, S., Melnyk, S., Savenka, A., Hubanks, A., Jernigan, S., Cleves, M., \& James, S. J. (2008). The frequency of polymorphisms affecting lead and mercury toxicity among children with autism. Am J Biochem Biotech, 4(2), 85-94

[26] Rossignol DA, Genuis SJ, Frye RE. Environmental toxicants and autism spectrum disorders: a systematic review. Transl Psychiatry 2014;4: e360.

[27] Schecter A, Pavuk M, Paepke O, Ryan JJ, Birnbaum L, Rosen R. Polybrominated diphenyl ethers (PBDEs) in U.S. mothers' milk. Environ. Health Perspect. 2003; 111(14):1723-1729. [PubMed: 14594622]

[28] Sealey, L. A., Hughes, B. W., Sriskanda, A. N., Guest, J. R., Gibson, A. D., Johnson-Williams, L., \& Bagasra, O. (2016). Environmental factors in the development of autism spectrum disorders. Environment international, 88, 288-298.

[29] Shelton, J. F., Hertz-Picciotto, I., \& Pessah, I. N. (2012). Tipping the balance of autism risk: potential mechanisms linking pesticides and autism. Environmental health perspectives, 120(7), 944-951.

[30] Sjodin A, Hagmar L, Klasson-Wehler E, Kronholm-Dlab K, Jakobsson E, Bergman O. Flame retardant exposure: Polybrominated diphenyl ethers in blood from Swedish workers. Environ. Health Perspect. 1999; 107(8):643-648. [PubMed: 10417362]

[31] Snow WM, Hartle K, Ivanco TL. Altered morphology of motor cortex neurons in the VPA rat model of autism. Dev Psychobiol 2008; 50:633-639. Report from a study using the valproic-acid rat model for autism. The study used the valproic-acid model to examine brain development and developmental injuries, possibly related to autism

[32] Theodora Stavridou, Anna Maria Driga, Athanasios Drigas ( Net Media Lab-Mind \& Brain R\&D, N.C.S.R. 'Demokritos', Athens, Greece dr@iit.demokritos.g Blood Markers in Detection of Autism https://doi.org/10.3991/ijes.v9i2.21283

[33] US Environmental Protection Agency. Toxic Release Inventory (TRI) Program. Washington, DC. http://www.epa.gov/tri/tridata/tri06/index.htm. [Accessed 12 December 2008]

[34] Van Wijngaarden E, Davidson PW, Smith TH, Evans K, Yost K, Love T, et al. Autism spectrum disorder phenotypes and prenatal exposure to methylmercury. Epidimiology 2013; 24(5): 651-9

[35] Volaki, K. (2012). Molecular study of patients with autistic behavior (Doctoral dissertation, National and Kapodistrian University of Athens (EKPA). School of Health Sciences. Department of Medicine. Department of Maternal and Child Health. Laboratory of Medical Genetics)

[36] Wong, S., \& Giulivi, C. (2016). Autism, mitochondria and polybrominated diphenyl ether exposure. CNS \& Neurological Disorders-Drug Targets (Formerly Current Drug Targets-CNS \& Neurological Disorders), 15(5), 614-623

[37] Zablotsky, B., Black, L.I., Maenner, M.J., Schieve, L.A., Blumberg, S.J., 2015. Estimated prevalence of autism and other developmental disabilities following questionnaire changes in the 2014 National Health Interview Survey. Natl. Health Stat. Rep. 87, 1-21 